\title{
Combinatorial Optimization of Resolution Level for 3D Scene Based on
}

\section{Regional Weighted Information Entropy}

\author{
Li CHE $^{1,2, a^{*}}, \quad$ Fengju KANG ${ }^{1,2, b}$
}

\author{
${ }^{1}$ School of Marine Science and Technology, Northwestern Polytechnical University, Xi'an , China \\ ${ }^{2}$ National Key Laboratory of Underwater Information Process and Control, Xi'an, China \\ anwpu_vr@163.com, bangfengju@nwpu.edu.cn
}

\begin{abstract}
Keywords: 3D scene; Resolution level; Visual perception; Information entropy; Particle Swarm Optimization

Abstract. With respect to the problem that traditional combination of resolution level methods for 3D scene either can easily fall into tedious and repeated experiments for interaction design or fail to provide results that conform to human visual habits. In this paper, an intelligent combination of resolution level based on visual perception driven visual quality evaluation method is proposed. Through analyzing the influence of several digital image factors on human visual characteristic, the mathematical models are established. Combined with information entropy theory, an objective image quality evaluation metric in line with visual characteristics is built. Evolution algorithm is utilized to select the resolution level automatically and intelligently. Experimental results demonstrate that the scene drawn by the proposed algorithm shares more common with the sensory choice of human beings. In comparison with existing methods, the proposed method has high efficiency and requires no user interaction.
\end{abstract}

\section{Introduction}

With the rapid development of 3D modeling and data capture technology, the complexity of 3D mesh model seems to grow faster than the ability of graphics hardware to render them interactively. Mesh simplification offers one solution [1], which can create multi-resolution model representation of the object in the scene and improve the rendering performance.

Variety types of 3D data and the increasing complexity of the scene, how to intuitive and efficient rendering of the scene gradually become a hot spot in 3D visualization technology. The first technique for choosing the LoD of polygonal objects have used static metrics such as visibility and distance [2], projection size [3] and the span of level changed [4]. These simple metrics improve the frame rate in many cases, but can not guarantee a regulated or bounded execution time. To guarantee a bounded frame rate, Funkhouser and Sequin [5] have presented a predictive technique that use an estimate of the execution time for the correct choice of the LoD to use. However, the greedy solution only guarantees to be half as good as the optimal solution. In recent years, image-based rendering have received a lot of interest because of its ability to represent complex models in a compact way. Researchers pay more attention on Geometric-image hybrid rendering strategy $[6,7]$. These algorithms can generate the best visual quality scene while meeting timing constraints. Artificial intelligence algorithms, such as PSO, support vector machines, random search algorithm, are applied in 3D visualization. In [8] the transfer function is designed automatically by PSO algorithm. In [9], GA-PSO algorithm is introduced to solve the multi-resolution model combination problem, different resolution model combination can be 
considered as a particle, the search for optimal combination is reformulated as a global optimization problem.

This paper presents a novel combination and optimization of resolution level of multiple models for 3D scene. Visual perception driven regional weighted information entropy, which combines human visual perception perception characteristics and image information entropy, is used to evaluate the visual quality of 3D scene. Different resolution level combination is considered as a particle. In order to overcome the problem that basic PSO algorithm can easily drop into local optimum, adaptive weight Particle Swarm Optimization(PSO) is utilized for the resolution level combinatorial optimization process.

The rest of this paper is organized as follows: Several visual perception influencing factors are given in section 1. The novel viewpoint quality evaluation metric named Region Weighted Information Entropy is proposed in detail in section 2. In section 3, Combinatorial Optimization of resolution level using adaptive weight PSO is presented. Experimental results and comparison studies to verify the capability of the proposed method are shown in section 4. Conclusion and future work are given in section 5 .

\section{Visual perception influencing factor}

From the perspective of visual psychology, the vision is a kind of positive feelings behavior, not only relates with the physiological factors, but also depends on psychological factors. When observing an image, people pay more attention on the region which has significant change, while the smooth region with uniform brightness or texture region with similar spatial frequency is ignored.

\section{Luminance influencing factor}

The luminance variance dominates the recognition of objects in a scene because a scene with low luminance (dark) provides difficult recognition of objects and a scene with high luminance (bright) is hard to extract the edges of objects. Therefore, a scene with wide range of luminance values, namely, with high contrast is considered as a good view. The Weber-Fechner rule [10] illustrates that subjective brightness of visual is proportional to the logarithm of light stimulus intensity. Luminance sensitive factor is defined as:

$$
L=K \times \log \frac{l^{\max }}{l^{\text {avg }}}=K \times \log \frac{\max \left\{Y_{1}, Y_{2}, Y_{3} \mathrm{~K} Y_{N}\right\}}{\frac{1}{N} \sum_{i=1}^{N} Y_{i}}
$$

Where $l^{\max }$ is the maximum luminance value of image, $l^{a v g}$ is average luminance value, $l^{\max } / l^{a v g}$ approximately expresses the image light stimulation intensity. $K$ usually sets 1 .

\section{Chrominance influencing factor}

Visualizing multi-dimensional data, we often focus a specific element of the array data by representing with color change. This is a good example of using chrominance property for good viewpoints. A higher chrominance total or its abrupt change seems to provide better views. We use chrominance variance to represent the impact on the human visual perception. Chrominance influencing factor is defined as:

$$
C=\lambda \times C_{I}+(1-\lambda) \times C_{Q}
$$

Where $C_{I}, C_{Q}$ is the chrominance variance of $I$ and $Q . \lambda$ is set $0 \sim 0.5$. 


$$
C_{I}=\sqrt{\frac{1}{N-1} \sum_{i=1}^{N}\left(I_{i}-\frac{1}{N} \sum_{i=1}^{N} I_{i}\right)} \quad C_{Q}=\sqrt{\frac{1}{N-1} \sum_{i=1}^{N}\left(Q_{i}-\frac{1}{N} \sum_{i=1}^{N} Q_{i}\right)}
$$

\section{Texture details influencing factor}

Spatial frequency indicates the clarity of image detail, we use spatial frequency as a measurement of the degree that texture detail influencing on human visual perception. The higher spatial frequency is, the more texture detail region contains, and the more attention human visual will be paid. Texture detail influencing factor is defined as:

$$
D(F)=\sqrt{R F^{2}+C F^{2}}
$$

Where $R F$ and $C F$ are the row and column frequency of the image pixels. Expressions are as follows.

$$
R F=\sqrt{\frac{1}{m \times n} \sum_{i=1}^{m-1} \sum_{j=1}^{n-1}(F(i, j)-F(i, j+1))^{2}} R F=\sqrt{\frac{1}{m \times n} \sum_{i=1}^{m-1} \sum_{j=1}^{n-1}(F(i, j)-F(i+1, j))^{2}}
$$

\section{Spatial location influencing factor}

Research on HVS has shown that when people observe a picture, the first note is the central part, and then turns around. Namely the importance of the image is generally decreasing from the center to the surrounding. Assuming that the image is divided into $n$ equal size blocks, and the spatial location influencing factor of the block $j$ is defined as:

$$
S_{j}=1-(1-\beta) \times \frac{\sqrt{\left(x_{j c}-x_{c}\right)^{2}-\left(y_{j c}-y_{c}\right)^{2}}}{d}
$$

Where $x_{c}$ and $y_{c}$ are the center coordinates of image $X . x_{j c}$ and $y_{j c}$ are the center coordinates of block $j . d$ represents the maximum distance that each point of original image to the center coordinates. $\beta$ is the basis weight and usually set $0 \sim 0.5$.

\section{Regional Weighted Information Entropy}

Information entropy (IE) used to measure the degree of information richness in an image, but not contains luminance, chrominance, texture details and other information. While, these factors have strong impact on the human visual perception, so we combine visual perception influencing factors with IE to make the image quality evaluation result more conform to human visual habits. We name the new criterion for scene image quality as Regional Weighted Information Entropy (RWIE). Figure 1 presents the implementation process of RWIE.

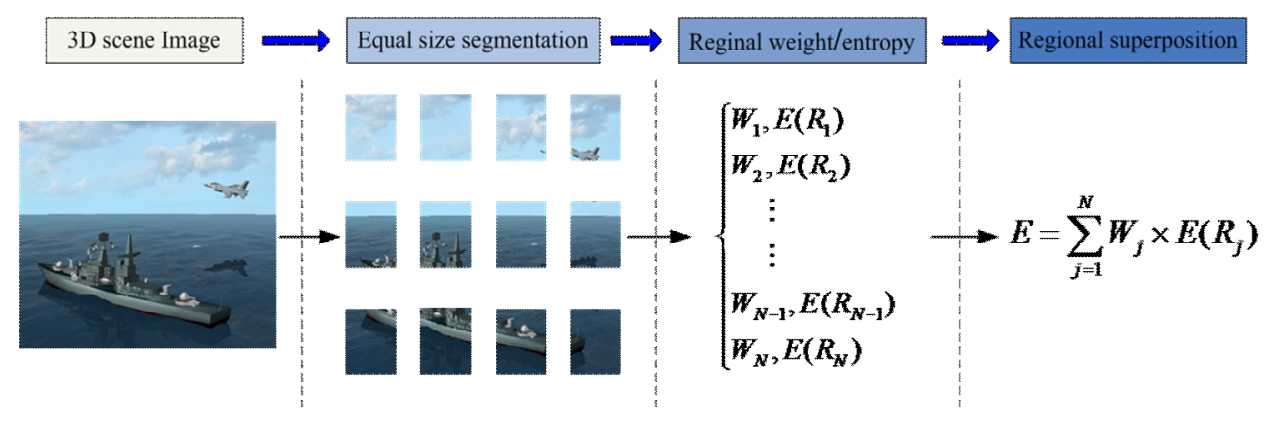

Figure 1 Implementation process of RWIE

Definition: Assume that image $X$ is divided into $N$ regions with equal size. Each region is defined as $R_{j}$ and contains $m \times n$ pixels. 


\section{Image information entropy}

John Zachary [11] introduced the concept of information entropy to the image processing. Color histogram of image is seen as the probability density function. Image information entropy is a statistical form of character, which reflects the average amount of information in the image, and the expression defines as follows:

$$
\begin{gathered}
E(I M G)=-\sum_{i=1}^{m} \sum_{j=1}^{n} p(i, j) \log _{2} p(i, j) \\
p(i, j)=x(i, j) / \sum_{i=1}^{m} \sum_{j=1}^{n} x(i, j)
\end{gathered}
$$

Where $m, n$ indicate the size of the image, $x(i, j)$ is the gray value at the point $(i, j)$.

\section{Image regional weight}

Computing the four visual perception influencing factors for each region, after normalization by $\mathrm{Eq}(9)$

$$
L_{j}^{\prime}=\frac{L_{j}}{\sum_{j=1}^{N} L_{j}} \quad C_{j}^{\prime}=\frac{C_{j}}{\sum_{j=1}^{N} C_{j}} \quad D_{j}^{\prime}=\frac{D_{j}}{\sum_{j=1}^{N} D_{j}} \quad S_{j}^{\prime}=\frac{S_{j}}{\sum_{j=1}^{N} S_{j}}
$$

The weight of region $j$ is defined as:

$$
W_{j}=L_{j}^{\prime}+C_{j}^{\prime}+D_{j}^{\prime}+S_{j}^{\prime}
$$

After the computation of regional image information entropy $E\left(R_{j}\right)$ and regional weight $W_{j}$, the Regional Weighted Information Entropy (RWIE) for the given viewpoint $V p i$ is defined as:

$$
E=\sum_{j=1}^{N} W_{j} \times E\left(R_{j}\right)
$$

According to Figure 1, the overall computational process of RWIE is showed by Algorithm 1.

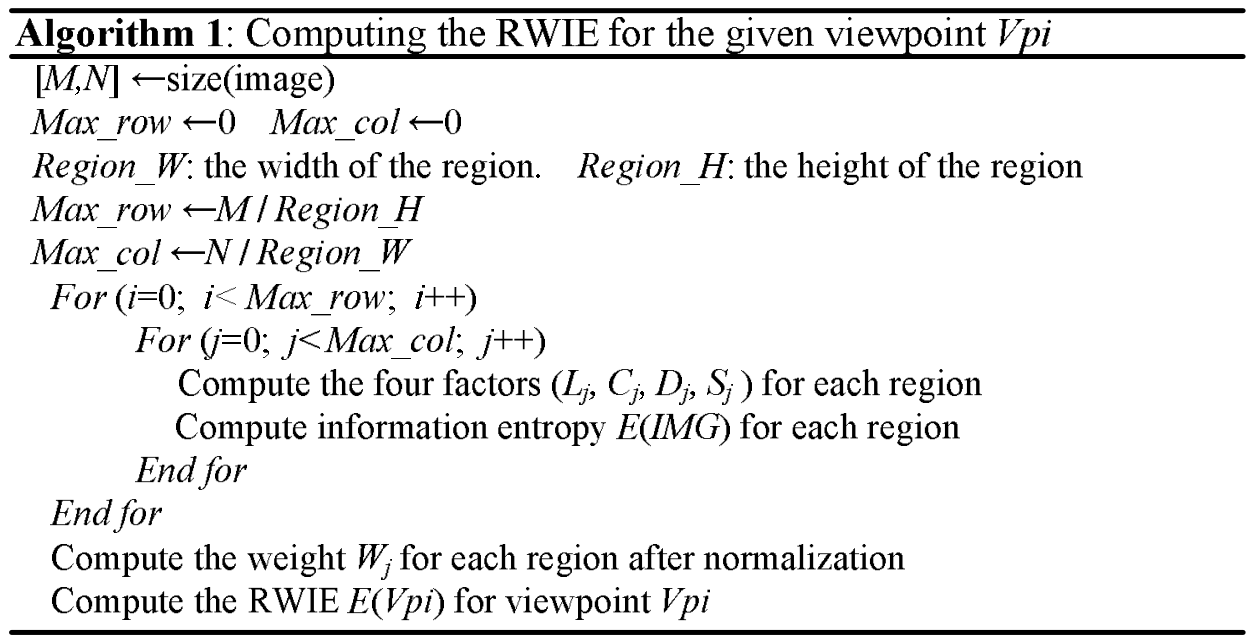

\section{Combinatorial Optimization using adaptive weight PSO}

In the process of search for optimal resolution level combination, resolution level combination is encoded as particles, From the initial combination sets, each combination is the particle position in the search space, the fitness value of each particle is determine by visual quality 
evaluation(RWIE). Velocity and position is updated by PSO. Through continuously iterative optimization satisfactory combination will be found.

we use adaptive weight PSO algorithm to overcome shortcoming that standard PSO algorithm can easily drop into local optimum. In modified PSO, velocity and position of each particle is updated by Eq (12) and Eq (13).

$$
\begin{gathered}
v_{i m}^{k+1}=w^{*} v_{i m}^{k}+c_{1} * \operatorname{Rand}() *\left(p_{i m}^{k}-x_{i m}^{k}\right)+c_{2} * \operatorname{Rand}() *\left(p_{g m}^{k}-x_{i m}^{k}\right) \\
x_{i m}^{k+1}=x_{i m}^{k}+v_{i m}^{k}
\end{gathered}
$$

$w$ is weight coefficient, which can improve global and local search ability of the algorithm. The adaptive weight is defined as:

$$
w=\left\{\begin{array}{l}
w_{\text {min }}-\frac{\left(w_{\max }-w_{\min }\right) *\left(f-f_{\min }\right)}{f_{\text {avg }}-f_{\min }}, f \leq f_{\text {avg }} \\
w_{\text {max }}, \quad f>f_{\text {avg }}
\end{array}\right.
$$

Where $w_{\min }$ and $w_{\max }$ represent the maximum and minimum value of weight, $f_{\text {avg }}$ and $f_{\min }$ are the average and minimum fitness value of all current particles. $f$ is the fitness value of current particle.

Algorithm 2 shows the overall procedure of resolution level combinatorial optimization. In each iteration step, the values of pBest and gBest are updated for every particle, where pBest is the best solution of the particle achieved so far, and gBest is the global best value obtained so far by all particles in the population.

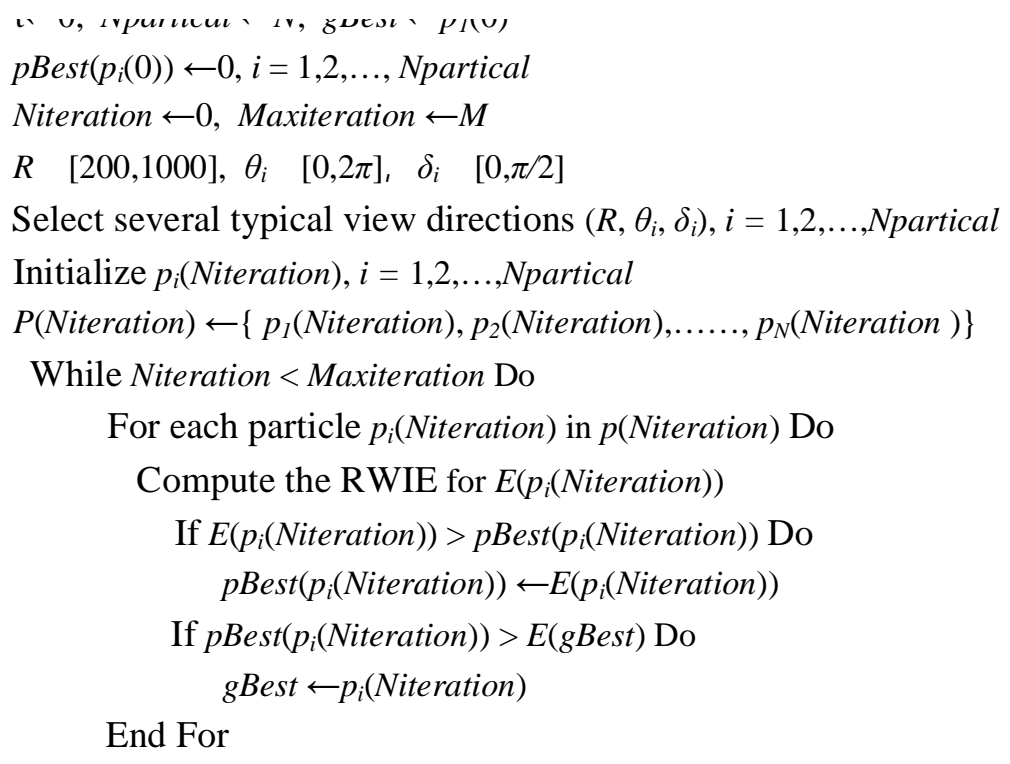

\section{Experimental Results and Analysis}

The proposed algorithm is carried on Intel Core i7 2.93GHZ with 4GB RAM and NVIDA GTX graphic card. Software configuration is Window 7 system, OpenGL 3.2, OSG 2.8.2, VS 2012. Test scene is formed by three models:aircraft, ship and sea, all the models have five resolution levels. Table. 1 shows the corresponding parameters setting of adaptive weight PSO in the process of optimal resolution level combination. 
Table 1. Parameters setting of adaptive weight PSO

\begin{tabular}{cccccc}
\hline Population size & Dimension & Maximum iterations number & $w_{\max }$ & $w_{\min }$ & $c_{1}, c_{2}$ \\
\hline 3 & 3 & 30 & 0.9 & 0.4 & 2 \\
\hline
\end{tabular}

Searching for the optimal resolution level combination by the adaptive weight PSO iterations eliminates reluctant viewpoint evaluations, and thus improves the efficiency of the searching process. We use the RWIE as the objective optimization function. Fig 2 shows the change of fitness value of each particle in the evolutionary process. The optimal resolution level combination is obtained by the second particle in the ninth generation.

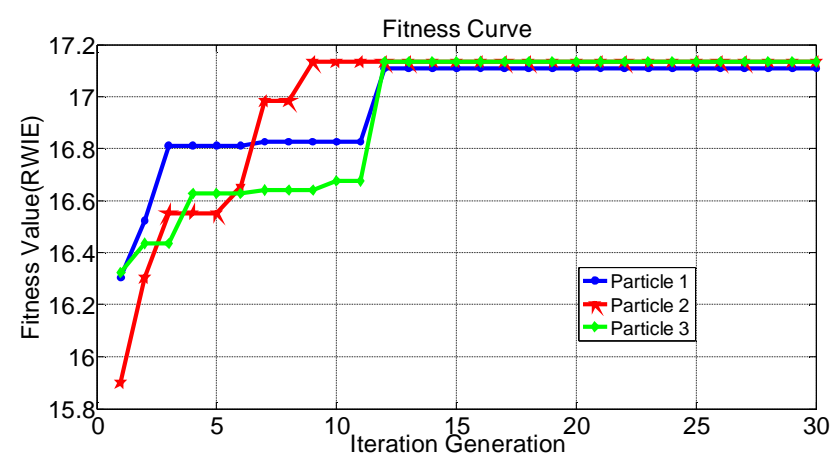

Fig 2 Fitness curve of three particles

We designed a comparative experiment. Fig 3 shows the change of fitness value of adaptive weight PSO and standard PSO in the process of search for optimal resolution level combination. Results show that adaptive weight PSO has higher convergence speed than standard PSO, the optimal resolution level combination can be obtained in about nine generations, while standard PSO needs eighteen generations.

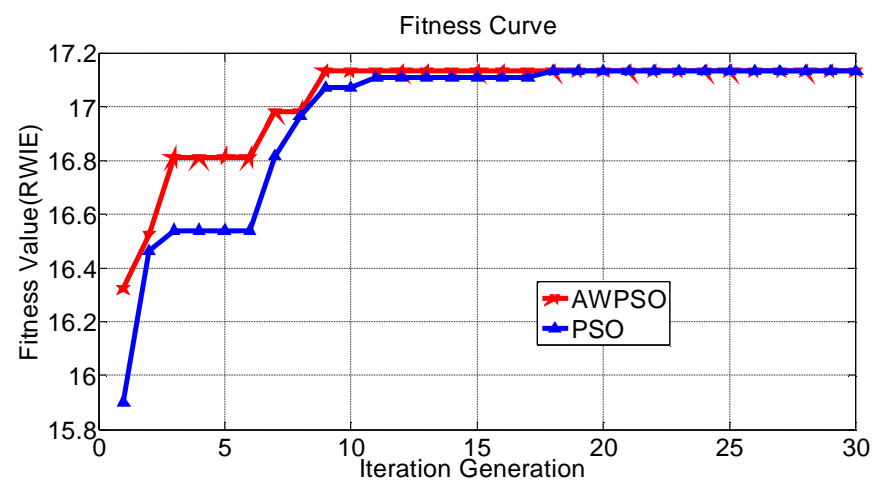

Fig 3 Fitness curve of adaptive weight PSO and standard PSO

The obtained optimal resolution level combination and the drawn image by [5], [9] and proposed algorithm are showed in Figure 4.

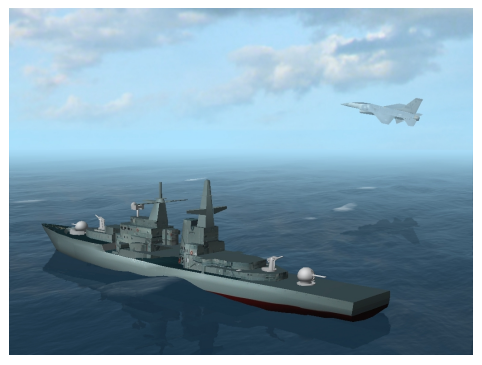

(a) algorithm in [5]

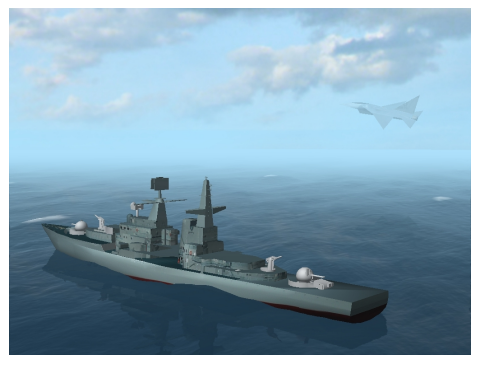

(b) algorithm in [9]

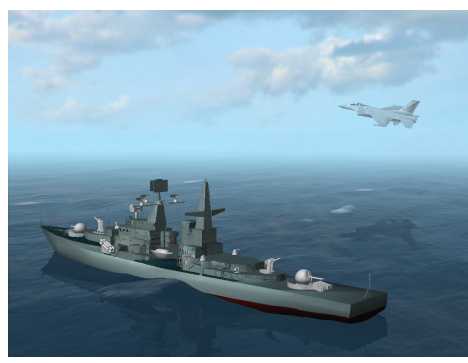

(c) proposed algorithm

Fig 4 Optimal scene rendered by algorithm in [5],[9]and proposed algorithm 
Image 4(a) is drawn by the algorithm in [5], the resolution level of sea, ship and aircraft is 4, 2, 3. Image 4(b) is drawn by the algorithm in [9], and the corresponding resolution level is 5, 3, 2. Image 4(c) is drawn by the proposed method, and the corresponding resolution level is 5, 4, 3. From the resolution level selected of three models and the visual quality evaluation result, we can see image (c) is better than other images. The corresponding resolution level obtained by the proposed method is more adapted to human visual perception characteristics that the near object we can see more clearly should use high resolution level, while far object use low resolution level.

\section{Conclusions and Future Work}

In this paper, we present four visual perception influencing factor, combined with the image information entropy, a new image quality evaluation metric which is more conform to human visual habits is proposed and applied to the optimal resolution level combination for 3D scene. Using PSO algorithm can effectively reduce the number of scene image evaluation and eliminates the redundant combination calculation, thus quickly obtain the optimal combination mode.

The frame rate, as one of the 3D scene real-time performance indicators, combine with image information entropy or proposed RWIE to select the optimal resolution level combination, maximize the visual effect and meet the time constrain will be our future work.

\section{References}

[1] Yaping Zhang, Hua Xiong. A Survey of Simplification and Multiresolution Techniques for Massive Meshes. Journal of Computer-Aided Design \& Computer Graphics,2010,22(4): 560-568 (In Chinese).

[2] CARLSON, D. HODGINS, J. Simulation levels of detail for real-time animation. In Proceedings-Graphics Interface, pp.1-8, 1997.

[3] Redon, Stephane. Galoppe, Nico. Lin, Ming C. Adaptive dynamics of articulated bodies. ACM Transactions on Graphics, 24(3), pp.936-945, 2005.

[4] Zhuo Yu. Xiaohui Liang. Zhiyu Chen. A Level of Detail Selection Method for Multi-Type Objects Based on The Span of Level Changed. IEEE International Conference on Robotics, Automation and Mechatronics, pp.1102-1107, 2008.

[5] Funkhouser, T. A. Sequin, C. H. Adaptive display algorithm for interactive frame rates during visualization of complex virtual environments. Computer Graphics, pp.247-254, 1993.

[6] Jehaes, T. Quax, P. Monsieurs. Hybrid representations to improve both streaming and rendering of dynamic networked virtual environments. In Proceedings of VRCAI, pp.26-32, 2004.

[7] Jehaes, Tom. Lamotte, Wim. Tack, Nicolaas. OBJECTIVE QUALITY SELECTION FOR HYBRID LOD MODELS. Proceedings of the 3rd International Conference on Computer Graphics Theory and Applications, pp.241-248, 2008.

[8] Yanni Wang, Yao Zheng. Intelligent Volume Visualization through Transfer Function and Viewpoint Selection. Journal of Computer-Aided Design \& Computer Graphics. 2008, 20(5): 565-570 (In Chinese).

[9] Li CHE, Fengju KANG. Intelligent Combination of Discrete LoD Model for 3D Visualization Based on Visual Perception and Information Entropy Fusion. Journal of System Simulation 2015,27(8):1815-1823. 
[10] Xuguang Lu, Yuefeng Wang, Wengang Hu, eta.l A method of image quality assessment based on visual regions of interest [J]. Microcomputer Information, 2005, 21(10-3): 95-96(In Chinese).

[11] John Z M. An information theoretic approach to content based image retrieval, Louisiana State University and Agricultural and Mechanical College, Ph.D, 2000. 